\title{
SOME RESULTS IN REPRESENTABLE BANACH SPACES
}

\author{
Miguel A. Canela
}

\begin{abstract}
Some results are presented, concerning a class of Banach spaces introduced by G. Godefroy and M. Talagrand, the representable Ban ach spaces. The main aspects considered here are the stability in forming tensor products, and the topological properties of the weak* dual unitball.
\end{abstract}

This note deals with some results on a class of Banach spaces introduced by G. Godefroy and M. Talagrand in $[\boldsymbol{7}]$, the representable Banach spaces. We begin by recalling some concepts.

First, a Hausdorff topological space is said to be analytic if it is a continuous image of the irrationals. A measurable space is said to be a Souslin space if it is Borel isomorphic to some space $(A, \Sigma)$, where $A$ is a subset of the real line and $\Sigma$ is the $\sigma$-algebra of the Borel subsets of $A$.

Let $X$ be a normed space, and $D$ a subset of its dual $X^{*} . D$ is said to be norming if

$$
x \longrightarrow \sup \left\{\left|x^{*}(x)\right|: x^{*} \in D\right\}
$$

defines a norm equivalent to the given one. If $X$ admits a countable norming set $D \subset X^{*}$, we say that $X$ is a space of countable type. In this case we can consider the topology $\sigma_{D}$ of pointwise convergence on the points of $D$, which is a metrizable topology on $X$. We say that $X$ is a representable normed space if there is a countable norming set $D \subset X^{*}$ such that $\left(X, \sigma_{D}\right)$ is analytic. If $\left(X, \sigma_{D}\right)$ is analytic for every such $D$, we say that $X$ is universally representable. These spaces have been introduced in [7], where some questions about them are discussed.

A Hausdorff topological space $E$ is said to be angelic when for every relatively countably compact subset $A \subset E$ the following holds: a) $A$ is relatively compact, and b) every closure point $x \in \bar{A}$ is the limit of a sequence contained in $A$. The fundamental facts about angelic spaces can be found in [5]. A remarkable class of angelic compact spaces, the Rosenthal compacts, has been studied by Godefroy in [6]. A compact Hausdorff space is said to be a Rosenthal compact when it is homeomorphic to a subset of $B_{1}(I)$, the space of first class functions 
on the irrationals, endowed with the topology of pointwise convergence. Such a compact space is angelic, according to a celebrated result of Bourgain, Fremlin and Talagrand (see [x]). If $K$ is a Hausdorff separable compact space, $C(K)$ is universally representable if and only if $K$ is a Rosenthal compact. This is equivalent to the fact that for every countable dense subset $D \subset K$, the uniformity induced by $K$ on $D$ is analytic as a subset of $\{0,1\}^{D \times D}([6$, Theorem 4]). Similar problems have been studied in $\{3\}$.

A. Gulisahvili ([8]) has considered the following property for a Banach space $X:$ If $(\Omega, \Sigma)$ is a measurable space and $f: \Omega \longrightarrow X$ is totally scalarly measurable, then $f$ is scalarly measurable. This property will be labelled as property D.

We start the exposition by the following result, which is easy to prove, and probably known to the reader:

Lemma 1. Let $X$ be a normed space. Then $X$ has countable type if and only if $X$ can be renormed in such a way that the closed dual unit ball is weak* separable.

Lemma 2. Let $X$ be a (universally) representable normed space, and $\hat{X}$ be its completion. Then $\hat{X}$ is a (universally) representable Banach space.

Proof: Take a countable norming set $D \subset X^{*}$. We can suppose that $\left(X, \sigma_{D}\right)$ is analytic and that $D$ is weak ${ }^{*}$ dense in $B_{X}$. The closed unit ball $B_{X}$ of $X$ is closed in $\left(X, \sigma_{D}\right)$, and hence analytic. The map $\psi: X \times\left(B_{X}\right)^{N} \longrightarrow \hat{X}$ defined by

$$
\left(x,\left\{x_{n}\right\}_{n}\right) \longrightarrow x+\sum_{n=1}^{\infty} 2^{-n} x_{n}
$$

is surjective and continuous for the topology $\sigma_{D}$. Therefore $\left(X, \sigma_{D}\right)$ is anaIytic.

If $A$ is a total subset of a Banach space $X$ and $D \subset X^{*}$ is a countable norming set such that $\left(A, \sigma_{D}\right)$ is analytic, the finear span of $A$ is analytic $([9$, V.1.A. $)$ and we can apply the preceding Lemma to obtain:

Corollary 3. If $X$ is a Banach space which admits a total subset $A \subset X$ and a countable norming set $D \subset X^{*}$ such that $\left(A, \sigma_{D}\right)$ is analytic, then $X$ is representable. If $\left(A, \sigma_{D}\right)$ is analytic for every such $D$ (A fixed), then $X$ is universally representable.

Corollary 4. Let $K$ be a separable Hausdorff compact space, and suppose that there is a subset $A \subset C(K)$ which separates the points of $K$ and such that $\left(A, \sigma_{D}\right)$ is analytic for every countable dense subset $D \subset K$. Then $D$ is a Rosenthal compact. 
Proof: The algebra generated by $A$ and the constant functions is dense by the Stone-Weierstrass theorem, and the preceding Corollary can be applied to this case. To check that this algebra is analytic for $\sigma_{D}$ is a routine.

We give now a characterization of the universally representable Banack spaces which extends Theorem 6 of [7]:

Proposition 5. Let $X$ be a representable Banach space. The following are equivalent:

(i) $X$ is universally representable.

(ii) $X$ does not contain any copy of $l_{1}([0,1])$.

(iii) $X^{*}$ is weak* angelic.

(iv) $B_{X}$. is weak sequentially compact.

(v) $B_{X}$ - is a Rosenthal compact for the weak* topology.

(vi) $X$ can be renormed in such a way that the closed dual unit ball is a separable Roshental compact for the weak ${ }^{*}$ topology.

(vii) $X$ has property $D$.

Proof: The first three assertions are equivalent by the Theorem 6 of $[7]$.

(i) $\Longrightarrow$ (vi) Renorm $X$ in a such a way that the closed dual unit ball is weak* separable, and take a countable dense subset $D$ in this ball. $\left(X, \sigma_{D}\right)$ is analytic, and the points of $D$ can be considered as continuous real functions on this space. By (iii), the points of the ball can be considered as real functions on $\left(X, \sigma_{D}\right)$ which are pointwise limits of sequences of continuous functions, i.e. functions of the first Baire class. $\left(X, \sigma_{D}\right)$ is a continuous image of the irrationals, and so the closed unit dual ball is homeomorphic, for the weak* topology, to a compact set of first Baire class functions on the irrationals.

(vi) $\Longrightarrow$ (v) Trivial.

(v) $\Longrightarrow$ (iii) By the result of $[\mathbf{1}]$ mentioned in tha introduction.

(iii) $\Longrightarrow$ (iv) Every compact angelic space is sequentially compact (see Chapter III of [5]).

(iii) $\Longrightarrow$ (vii) It is a result of Gulisashvili ([8]).

(vii) $\Longrightarrow$ (ii) Closed subspaces obviously inherit property $D$, and Gulisashvili has shown that $l_{1}([0,1])$ does not have the property $D$.

(iv) $\Longrightarrow$ (ii) If $l_{1}([0,1]) \subset X$, the unit ball of $l_{\infty}([0,1])$ is contained in a weak ${ }^{*}$-weak ${ }^{*}$ continuous image of $B_{X}$.

The following Corollaries can be easily obtained from Proposition 5:

Corollary 6. Let $X$ be a universally representable Banach space, and $Y \subset X$ a closed subspace which is a representable Banach space. Then $Y$ is universally representable.

Corollary 7. Let $K$ be a separable Rosenthal compact and $K^{\prime}$ a representable compact space, and suppose that there exists a continuous surjection $f: K \longrightarrow K^{\prime}$. Then $K^{\prime}$ is a Rosenthal compact. 
Remark. Godefroy $([6])$ has constructed an example of a separable Rosenthal compact with a continuous image which is not representable. Thus, the assumption of the representability of $Y$ in Corollary 6 is not superfluous.

Corollary 8. Let $X, Y$ be representable Banach spaces, and suppose that there is a bounded linear operator $T: X \rightarrow Y$ with dense range. If $X$ is universally representable, then $Y$ is universally representable.

Proof: $T^{*}: Y^{*} \longrightarrow X^{*}$ is one-one and weak ${ }^{*}$-weak ${ }^{*}$ continuous. It suffices to use condition (iii) in Proposition 5 and the angelic lemma.

If $X$ is a Banach space, we denote by $C y l(X)$ the cylindrical $\sigma$ - algebra on $X$, generated by the functionals of $X^{*}$, which is known to coincide with the Baire $\sigma$-algebra of $(X, w e a k)$ ([4, theorem 2.3]). If $D \subset X^{*}$ is a countable norming set, we can suppose that $D$ is weak* dense in the closed unit ball of $X^{*}$ for an equivalent norm on $X$. Thus we can reproduce the argument of $[6$, Theorem 9] to obtain:

Proposition 9. Let $X$ be a Banach space of countable type. The following are equivalent:

(i) $X$ is universally representable.

(ii) $(X, C y l(X))$ is a Souslin measurable space.

A statement which is analogous to (i) $\Longrightarrow$ (vi) of Proposition 5 can be made for representable Banach spaces:

Proposition 10. Let $X$ be a Banach space of countable type. The following are equivalent:

(i) $X$ is representable.

(ii) $X$ can be renormed in such a way that the closed unit ball of $X^{*}$ admits, for the weak* topology, a dense countable subset on which it induces an analytic uniformity.

Proof: (i) $\Longrightarrow$ (ii) Let $D \subset X^{*}$ be a countable norming set, with $\left(X, \sigma_{D}\right)$ analytic. Replacing $D$ by its rational absolutely convex hull does not change the topology $\sigma_{D}$, and we can renorm $X$ in such a way that $D$ is weak ${ }^{*}$ dense in the ball $B_{X} \cdot$. Consider now the map

$$
\psi:\left(X, \sigma_{D}\right) \times N \longrightarrow\{0,1\}^{D \times D}
$$

defined by

$$
\left.\psi(x, k)=\left\{x^{*}, y^{*}\right) \in D \times D:\left|x^{*}(x)-y^{*}(x)\right| \leq k^{-1}\right\}
$$

whose range is a sub-base of the uniformity induced by $\left(B_{X} \cdot\right.$, weak $\left.{ }^{*}\right)$ on $D$. By means of the methods of $[3]$ the analyticity of this uniformity can be obtained, 
remarking that $\psi$ is a Borel map. (ii) $\Longrightarrow$ (i) Suppose $X$ renormed in such a way. By virtue of $[6$, lemma 2$], C\left(\left(B_{X *}\right.\right.$, weak $\left.\left.{ }^{*}\right)\right)$ is $\sigma_{D}$-analytic. It is easy to see that $\left(B_{X}, \sigma_{D}\right)$ is homeomorphic to a closed subset of this analytic space, and hence $\left(X, \sigma_{D}\right)$ is analytic.

Remark. Is the converse implication valid? If the answer is yes, it is easy to check that the assumption on the representability of $X$ is not necessary for the implication (vi) $\Longrightarrow$ (i) of Proposition 5 .

Now, we state our main result:

Proposition 11, Let $X, Y$ be Banach spaces. Then:

(i) If $X, Y$ both have countable type, $X \hat{\otimes}_{\epsilon} Y$ has countable type.

(ii) If $X, Y$ are representable, $X \hat{\otimes}_{\epsilon} Y$ is representable, and it is universally representable if and only if $X, Y$ are both universally representable.

Proof: (i) By Lemma 1, we can suppose that $B_{X}$, and $B_{Y}$. admit subsets $D \subset B_{X} \cdot, E \subset B_{X}$. which are countable and weak* dense. The identity

$$
\begin{gathered}
\sup \left\{\left|<x^{*} \otimes y^{*}, z>\right|: x^{*} \in D, y^{*} \in D, y^{*} \in E\right\}= \\
=\sup \left\{\left|<x^{*} \otimes y^{*}, z>\right|:\left\|x^{*}\right\| \leq 1,\left\|y^{*}\right\| \leq 1\right\}
\end{gathered}
$$

shows us that the set $G=D \otimes E$ is norming for the $\epsilon$ topology in $X \otimes Y$.

(ii) By Corollary 3 , it suffices to prove the existence of a conntable norming set $G \subset\left(X \otimes_{\epsilon} Y\right)^{*}$ and a total subset $A \subset X \otimes Y$ such that $\left(A, \sigma_{G}\right)$ is analytic. We take $D, E$, as in (i), with $\left(X, \sigma_{D}\right),\left(Y, \sigma_{E}\right)$ analytic, and we put $G=D \otimes E$. Actually, we only need to remark that the canonical bilinear map $\chi: X \times Y \rightarrow$ $X \otimes Y$ defined by $\chi(x, y)=x \otimes y$ is ( $\left.\sigma_{D} \times \sigma_{E}, \sigma_{G}\right)$-continuous.

Suppose that $B_{X}$. and $B_{Y}$. are separable Rosenthal compacts for the weak* topology. Then $B_{X} \times B_{Y}$ is a separable Rosential compact for the product topology, and $C\left(B_{X} \times B_{Y^{*}}\right)$ is universally representable. We finish by recalling that $X \hat{\otimes}_{\epsilon} Y$ is isometrically isomorphic to a closed subspace of $C\left(B_{X} \cdot \times B_{Y}\right)$ and by using Corollary 6 . The only if assertion follows directly from Corollary 6.

The representation of a space of vector-valued continuous functions on a compact space as an $\epsilon$ tensor product gives us directly:

Corollary 12. Let $K$ be a separable compact Hausdorff space, and $X$ a Banach space. Then:

(i) If $K$ has countable type, $C(K, X)$ has countable type.

(ii) If $K, X$ are representable, $C(K, X)$ is representable, and it is universally representable if and only if $K$ is a Rosenthal compact and $X$ is representable.

Finally, we give a result for operator spaces: 
Proposition 13. Let $X$ be a separable Banach space, $Y$ a Banach space, and $B(X, Y)$ the space of bounded linear operators of $X$ into $Y$. Then:

(i) If $Y$ has countable type, $B(X, Y)$ has countable type.

(ii) If $Y$ is representable, $B(X, Y)$ is representable.

Proof: (i) Let $D \subset B_{X}$ be a countable dense subset, and suppose that there is a subset $E \subset B_{Y}$. which is countable and weak ${ }^{*}$ dense. Each pair $\left(x, y^{*}\right) \in$ $\in D \times E$ defines an element of the unit ball $B_{B(X, Y)}$. through $T \longmapsto y^{*}(T x)$, and it is easy to see that the set $G$ of these elements is norming for $B(X, Y)$.

(ii) Take $D, E$ as above, and suppose that $\left(Y, \sigma_{D}\right)$ is analytic. Define $G$ as above and consider the map $T \longmapsto\{T x\}_{x \in D}$ which is a homeomorphism of $B_{B(X, Y)}$ onto a subset of $Y^{D}$ when $B_{B(X, Y)}$ is endowed with the topology $\sigma_{D}$ and $Y$ with $\sigma_{E}$. It suffices to see that this subset is closed. For this, take a sequence $\left\{T_{n}\right\}_{n}$ in $B_{B(X, Y)}$ and suppose that a map $T$ satisfies

$$
\sigma_{E}-\lim _{n \rightarrow \infty} T_{n} x=T x
$$

for every $x \in D$. E separates the points of $Y$, and we can extend $T$ to a linear operator defined on the linear span $X_{0}$ of $D$, such that the preceding relation holds for $x \in X_{0}$. Then $T\left(B_{X_{0}}\right) \subset B_{Y}$.

\section{References}

1. J. Bourgain, D.H. Fremlin and M. Talagrand, Pointwise compact sets of Baire-measurable functions, Amer. J. Math. 100 (1978), 845-886.

2. M. A. CANELA, Some sequential properties of the weak ${ }^{*}$ dual of a Banach space, Studia Univ. Babes-Bolyat 29 (1984), 29-32.

3. M. A. Canela, K-analytic uniform structures, Proc. Royal Soc. Edinb. $99(1984), 163-170$.

4. G. A. EDGAR, Measurability in a Banach space, Indiana Univ. Math. J. $26(1976), 663-677$.

5. G. Goderroy, Compacts de Rosenthal, Pacific J. Math. 91 (1980), 293-306.

7. G. GODEFROY AND M. TALAGRaND, Espaces de Banach représentables, Israel J. Math. 41 (1982), 321-330.

8. A. GJLISASHVILI, Estimates for the Pettis integral in interpolation spaces and inversion of the embedding theorems (in Russian), Dokl. Akad. Nauk SSSR 263 (1982), 788-793. 
9. J. HOFMANN-JORGENSEN., Various Publications Series, Aarhus Univ., Aarhus 10 (1970.), "The theory of analytic spaces,".

Departament de Matemàtica Aplicada i Anàlisi

Universitat de Barcelona

Barcelona 08071, SPAIN.

Rebrt el 4 d'Abrit de 1987 